Journal of Mathematics and Informatics

Vol. 11, 2017, 1-11

ISSN: 2349-0632 (P), 2349-0640 (online)

Published 11 December 2017

www.researchmathsci.org

DOI: http://dx.doi.org/10.22457/jmi.v11a1

Journal of

Mathematics and

Informatics

\title{
On the Cubic Diophantine Equation with Four Unknowns $\mathbf{x}^{2}+\mathbf{y}^{2}=z^{3}-w^{3}$
}

\author{
A.Vijayasankar ${ }^{1}$, M.A.Gopalan ${ }^{2}$ and V.Krithika ${ }^{3}$ \\ 1,3Department of Mathematics: National College, Trichy-620001 \\ Tamilnadu, India. e-mail;avsankar70@ yahoo.com \\ 1Department of Mathematics, SIGC, Trichy-620002 \\ Tamilnadu, India. \\ e-mail: mayilgopalan@gmail.com
}

Received 15 November 2017; accepted 4 December 2017

Abstract. The sequences of integral solutions to the cubic equation with four variables $x^{2}+y^{2}=z^{3}-w^{3}$ are obtained. A few properties among the solutions are presented. Also, Employing the integer solutions of the considered equation, integer solutions for different choices of hyperbolas and parabolas are obtained.

Keywords: Cubic equation with four unknowns, non-homogeneous cubic, cubic with four unknowns, integral solutions.

\section{AMS Mathematics Subject Classification (2010): 11D25}

\section{Introduction}

The Diophantine equation offers an unlimited field for research due to their variety [1-4]. In particular, one may refer [5-13] for cubic equation with three unknowns. In [14,15] cubic equations with four unknowns are studied for its non-trivial integral solutions and in $[16,17]$ cubic equations with five unknowns and one may refer $[18,19]$ for cubic equation with six unknowns are analyzed for its distinct integer solutions.

This communication concerns with the problem of obtaining infinitely many nonzero distinct integral solutions of cubic equation with four variables given by $x^{2}+y^{2}=z^{3}-w^{3}$. A few interesting properties among the solutions are presented.

\section{Method of analysis}

The cubic Diophantine equation with four unknowns to be solved

for getting non-zero integral solution is

$$
x^{2}+y^{2}=z^{3}-w^{3}
$$

To start with, it is observed by trial and error that the following quadruples

$$
(x, y, z, w): \quad(5,1,3,1), \quad(6,1,4,3), \quad(5,6,5,4), \quad(6,9,5,2), \quad(10,14,8,6),
$$

$\left(-\left(\alpha^{2}+1\right), \alpha\left(\alpha^{2}+1\right), 0,-\left(\alpha^{2}+1\right)\right)$ satisfy (1). 


\section{M.A.Gopalan and V.Krithika}

To obtain an infinite set of non-zero distinct integer solutions to (1), we proceed as follows:

On substituting the linear transformations

$$
z=x+h, w=x+k, h \neq k \neq 0
$$

in (1) it leads to

$$
x^{2}+y^{2}=(3 h-3 k) x^{2}+\left(3 h^{2}-3 k^{2}\right) x+h^{3}-k^{3}, h \neq k
$$

To Solve (3), we have to go in for particular values for $\mathrm{h}$ and $\mathrm{k}$. for simplicity and brevity, we present below a few illustrations when $\mathrm{h}$ and $\mathrm{k}$ take special values.

\subsection{Illustration 1}

Choose $h=k+1$ in the above equation We have

$$
y^{2}=2 x^{2}+3 x(2 k+1)+\left(3 k^{2}+3 k+1\right)
$$

for which the solutions are presented below when $k=1,2$

Case (1):

By taking $k=1$ in (4), we obtain

$$
y^{2}=2 x^{2}+9 x+7
$$

Performing some algebraic simplifications, the above equation is written as

$$
X^{2}=8 y^{2}+25
$$

where $X=4 x+9$

The smallest positive integer solution of (5) is

$$
X_{0}=15, y_{0}=5
$$

To obtain the other solutions of (5), consider the pell equation

$$
X^{2}=8 y^{2}+1
$$

whose general solution is given by

where

$$
\begin{aligned}
& \tilde{y}_{n}=\frac{g_{n}}{4 \sqrt{2}} \\
& \tilde{X}_{n}=\frac{f_{n}}{2} \\
& f_{n}=(3+2 \sqrt{2})^{n+1}+(3-2 \sqrt{2})^{n+1} \\
& g_{n}=(3+2 \sqrt{2})^{n+1}-(3-2 \sqrt{2})^{n+1}, n=0,2,4, \ldots
\end{aligned}
$$

Applying Brahmagupta lemma between $\left(X_{0}, y_{0}\right)$ and $\left(\tilde{X}_{n}, \tilde{y}_{n}\right)$, the other integer solutions of (5) are given by

$$
\begin{aligned}
& X_{n+1}=\frac{5}{2}\left[3 f_{n}+2 \sqrt{2} g_{n}\right] \\
& y_{n+1}=\frac{5}{4 \sqrt{2}}\left[2 \sqrt{2} f_{n}+3 g_{n}\right]
\end{aligned}
$$

By using (9) in (6) and using (2), we obtain the non-zero distinct integral solutions to (1) are given by 
On the Cubic Diophantine Equation with Four Unknowns

$$
\begin{aligned}
& x_{n+1}=\frac{5\left[3 f_{n}+2 \sqrt{2} g_{n}\right]-18}{8} \\
& y_{n+1}=\frac{5}{4 \sqrt{2}}\left[2 \sqrt{2} f_{n}+3 g_{n}\right] \\
& z_{n+1}=\frac{5\left[3 f_{n}+2 \sqrt{2} g_{n}\right]-2}{8} \\
& w_{n+1}=\frac{5\left[3 f_{n}+2 \sqrt{2} g_{n}\right]-10}{8}, n=0,2,4 \ldots
\end{aligned}
$$

The recurrence relations satisfied by $x, y, z$ and $w$ are given by

$$
\begin{aligned}
& x_{n+5}-34 x_{n+3}+x_{n+1}=72 . \\
& y_{n+5}-34 y_{n+3}+y_{n+1}=0 . \\
& z_{n+5}-34 z_{n+3}+z_{n+1}=8 . \\
& w_{n+5}-34 w_{n+3}+w_{n+1}=40, n=0,2,4, \ldots
\end{aligned}
$$

A few numerical examples are given below in Table 1:

Table 1 : Numerical examples

\begin{tabular}{|l|l|l|l|l|}
\hline $\mathrm{n}$ & $x_{n+1}$ & $y_{n+1}$ & $z_{n+1}$ & $w_{n+1}$ \\
\hline 0 & 19 & 30 & 21 & 20 \\
\hline 2 & 719 & 1020 & 721 & 720 \\
\hline 4 & 24499 & 34650 & 24501 & 24500 \\
\hline 6 & 832319 & 1177080 & 832321 & 832320 \\
\hline 8 & 28274419 & 39986070 & 28274421 & 28274420 \\
\hline
\end{tabular}

From the above table, we observe some interesting relations among the solutions which are presented below:

1. $x_{n+1}$ and $z_{n+1}$ are always odd

2. $y_{n+1}$ and $w_{n+1}$ are always even

3. Relations among the solutions:

$x_{n+5}=34 x_{n+3}-x_{n+1}+72$

$12 y_{n+1}=x_{n+3}-17 x_{n+1}-36$

$12 y_{n+3}=17 x_{n+3}-x_{n+1}+36$

$12 y_{n+5}=577 x_{n+3}-17 x_{n+1}+1260$ 
M.A.Gopalan and V.Krithika

* $12 y_{n+1}=17 x_{n+5}-577 x_{n+3}-1260$

$60 y_{n+3}=1331663 x_{n+3}-39199 x_{n+5}+2908044$

$12 y_{n+5}=17 x_{n+5}-x_{n+3}+36$

$577 y_{n+3}=24 x_{n+5}+17 y_{n+1}+54$

$577 y_{n+5}=816 x_{n+5}+y_{n+1}+1836$

$y_{n+1}=34 y_{n+3}-y_{n+5}$

Each of the following expressions represents a nasty number:

$$
\begin{aligned}
& >\frac{4}{5}\left\{70 x_{2 n+2}-2 x_{2 n+4}+168\right\} \\
& >\frac{4}{5}\left\{2378 x_{2 n+4}-70 x_{2 n+6}+5208\right\} \\
& >\frac{12}{2885}\left\{12 x_{2 n+6}-9512 y_{2 n+2}+2912\right\} \\
& >\frac{2}{5}\left\{99 y_{2 n+6}-3363 y_{2 n+4}+30\right\}
\end{aligned}
$$

Each of the following expressions in Table 2 represents a hyperbola:

Table 2: Hyperbola

\begin{tabular}{|l|l|}
\hline Hyperbola & $\left(p_{n} q_{n}\right)$ \\
\hline $2 p_{n}^{2}-9 q_{n}^{2}=450$ & $\left(70 x_{n+1}-2 x_{n+3}+153,33 x_{n+1}-x_{n+3}+72\right)$ \\
\hline $2 p_{n}^{2}-q_{n}^{2}=450$ & $\left(2378 x_{n+3}-70 x_{n+5}+5193,3363 x_{n+3}-99 x_{n+5}+7344\right)$ \\
\hline$p_{n}^{2}-8 q_{n}^{2}=8323225$ & $\left(12 x_{n+5}-9512 y_{n+1}+27,4 x_{n+5}-3363 y_{n+1}+9\right)$ \\
\hline$p_{n}^{2}-2 q_{n}^{2}=900$ & $\left(3363 y_{n+3}-99 y_{n+5}, 2378 y_{n+3}-70 y_{n+5}\right)$ \\
\hline
\end{tabular}

Each of the following expressions in Table 3 represents a parabola: 
On the Cubic Diophantine Equation with Four Unknowns

Table 3: Parabola

\begin{tabular}{|l|l|}
\hline Parabola & $\left(p_{n}, q_{n}\right)$ \\
\hline $3 q_{n}^{2}=5 p_{n}-150$ & $\left(70 x_{2 n+2}-2 x_{2 n+4}+168,33 x_{n+1}-x_{n+3}+72\right)$ \\
\hline$q_{n}^{2}=15 p_{n}-450$ & $\left(2378 x_{2 n+4}-70 x_{2 n+6}+5208,3363 x_{n+3}-99 x_{n+5}+\right.$ \\
\hline $16 q_{n}^{2}=2885 p_{n}-16646450$ & $\left(12 x_{2 n+6}-9512 y_{2 n+2}+2912,4 x_{n+5}-3363 y_{n+1}+9\right.$ \\
\hline $2 q_{n}^{2}=15 p_{n}-900$ & $\left(99 y_{2 n+6}-3363 y_{2 n+4}+30,2378 y_{n+3}-70 y_{n+5}\right)$ \\
\hline
\end{tabular}

Case (2):

By taking $k=2$ in (4), we obtain

$$
y^{2}=2 x^{2}+15 x+19
$$

Performing some algebraic simplifications, the above equation is written as

$$
\alpha^{2}=8 y^{2}+73
$$

where $\alpha=4 x+15$

The smallest positive integer solution of (10) is

$$
\alpha_{0}=9, y_{0}=1
$$

To obtain the other solutions of (10), consider the pell equation

$$
\alpha^{2}=8 y^{2}+1
$$

whose general solution is given by

where

$$
\begin{aligned}
& \tilde{y}_{n}=\frac{g_{n}}{4 \sqrt{2}} \\
& \tilde{\alpha}_{n}=\frac{f_{n}}{2} \\
& f_{n}=(3+2 \sqrt{2})^{n+1}+(3-2 \sqrt{2})^{n+1} \\
& g_{n}=(3+2 \sqrt{2})^{n+1}-(3-2 \sqrt{2})^{n+1}, n=0,2,4, \ldots
\end{aligned}
$$

Applying Brahmagupta lemma between $\left(X_{0}, y_{0}\right)$ and $\left(\tilde{X}_{n}, \tilde{y}_{n}\right)$, the other integer solutions of (10) are given by

$$
\begin{aligned}
& \alpha_{n+1}=\frac{1}{2}\left[9 f_{n}+2 \sqrt{2} g_{n}\right] \\
& y_{n+1}=\frac{1}{4 \sqrt{2}}\left[2 \sqrt{2} f_{n}+9 g_{n}\right]
\end{aligned}
$$

By using (14) in (11) and using (2), we obtain the non-zero distinct integral solutions to (1) are given by 
M.A.Gopalan and V.Krithika

$$
\begin{aligned}
& x_{n+1}=\frac{9 f_{n}+2 \sqrt{2} g_{n}-30}{8} \\
& y_{n+1}=\frac{1}{4 \sqrt{2}}\left[2 \sqrt{2} f_{n}+9 g_{n}\right] \\
& z_{n+1}=\frac{9 f_{n}+2 \sqrt{2} g_{n}-6}{8} \\
& w_{n+1}=\frac{9 f_{n}+2 \sqrt{2} g_{n}-14}{8}, n=0,2,4 \ldots
\end{aligned}
$$

The recurrence relations satisfied by $x, y, z$ and $w$ are given by

$$
\begin{aligned}
& x_{n+5}-34 x_{n+3}+x_{n+1}=72 . \\
& y_{n+5}-34 y_{n+3}+y_{n+1}=0 . \\
& z_{n+5}-34 z_{n+3}+z_{n+1}=24 . \\
& w_{n+5}-34 w_{n+3}+w_{n+1}=56, n=0,2,4, \ldots
\end{aligned}
$$

A few numerical examples are given below in Table 4:

Table 4: Numerical examples

\begin{tabular}{|l|l|l|l|l|}
\hline $\mathrm{n}$ & $x_{n+1}$ & $y_{n+1}$ & $z_{n+1}$ & $w_{n+1}$ \\
\hline 0 & 5 & 12 & 8 & 7 \\
\hline 2 & 289 & 414 & 292 & 291 \\
\hline 4 & 9941 & 14064 & 9944 & 9943 \\
\hline 6 & 337825 & 477762 & 337828 & 337827 \\
\hline 8 & 11476229 & 16229844 & 11476232 & 11476231 \\
\hline
\end{tabular}

From the above table, we observe some interesting relations among the solutions which are presented below:

1. $x_{n+1}$ and $z_{n+1}$ are always odd

2. $y_{n+1}$ and $w_{n+1}$ are always even

3. Relations among the solutions:

$$
\begin{aligned}
& x_{n+5}=34 x_{n+3}-x_{n+1}+120 \\
& * 12 y_{n+1}=x_{n+3}-17 x_{n+1}-60 \\
& * 12 y_{n+3}=17 x_{n+3}-x_{n+1}+60 \\
& * 1752 y_{n+5}=84242 x_{n+3}-2482 x_{n+1}+306600
\end{aligned}
$$


On the Cubic Diophantine Equation with Four Unknowns

$$
\begin{aligned}
& * 1752 y_{n+1}=2482 x_{n+5}-84242 x_{n+3}-306600 \\
& * 12 y_{n+3}=x_{n+5}-17 x_{n+3}-60 \\
& * 12 y_{n+5}=17 x_{n+5}-x_{n+3}+60 \\
& * 42121 y_{n+3}=1752 x_{n+5}+1241 y_{n+1}+6570 \\
& * 577 y_{n+5}=816 x_{n+5}+y_{n+1}+3060 \\
& * y_{n+1}=34 y_{n+3}-y_{n+5}
\end{aligned}
$$

Each of the following expressions represents a nasty number:

$$
\begin{aligned}
& >\frac{1}{73}\left\{568 x_{2 n+2}-8 x_{2 n+4}+2976\right\} \\
& >\frac{4}{73}\left\{4826 x_{2 n+4}-142 x_{2 n+6}+17784\right\} \\
& >\frac{2}{42121}\left\{36 x_{2 n+6}-19304 y_{2 n+2}+42256\right\} \\
& >\frac{2}{73}\left\{9 y_{2 n+4}-201 y_{2 n+2}+438\right\}
\end{aligned}
$$

Each of the following expressions in Table 5 represents a hyperbola:

Table 5: Hyperbola

\begin{tabular}{|l|l|}
\hline Hyperbola & $\left(p_{n}, q_{n}\right)$ \\
\hline$p_{n}^{2}-8 q_{n}^{2}=767376$ & $\left(568 x_{n+1}-8 x_{n+3}+2100,201 x_{n+1}-9 x_{n+3}+720\right)$ \\
\hline $2 p_{n}^{2}-q_{n}^{2}=95922$ & $\left(4826 x_{n+3}-142 x_{n+5}+17565,6825 x_{n+3}-201 x_{n+5}+2484\right.$ \\
\hline$p_{n}^{2}-8 q_{n}^{2}=1$ & $\left(36 x_{n+5}-19304 y_{n+1}+15,4 x_{n+5}-6825 y_{n+1}+15\right)$ \\
\hline$p_{n}^{2}-8 q_{n}^{2}=191844$ & $\left(201 y_{n+1}-9 y_{n+3}, 71 y_{n+1}-y_{n+3}\right)$ \\
\hline
\end{tabular}

Each of the following expressions in Table 6 represents a parabola: 
M.A.Gopalan and V.Krithika

Table 6: Parabola

\begin{tabular}{|l|l|}
\hline Parabola & $\left(p_{n}, q_{n}\right)$ \\
\hline $4 q_{n}^{2}=219 p_{n}-383688$ & $\left(568 x_{2 n+2}-8 x_{2 n+4}+2976,201 x_{n+1}-9 x_{n+3}+720\right.$ \\
\hline $4 q_{n}^{2}=876 p_{n}-383688$ & $\left(4826 x_{2 n+4}-142 x_{2 n+6}+17784,6825 x_{n+3}-201 x_{n}\right.$ \\
\hline $16 q_{n}^{2}=42121 p_{n}-35483572$ & $\left(36 x_{2 n+6}-19304 y_{2 n+2}+42256,4 x_{n+5}-6825 y_{n+1}\right.$ \\
\hline $8 q_{n}^{2}=219 p_{n}-191844$ & $\left(9 y_{2 n+4}-201 y_{2 n+2}+438,71 y_{n+1}-y_{n+3}\right)$ \\
\hline
\end{tabular}

\subsection{Illustration 2}

By taking $k=0, h=1$ in (4), we obtain

$$
y^{2}=2 x^{2}+3 x+1
$$

Performing some algebraic simplifications, the above equation is written as

$$
\beta^{2}=8 y^{2}+1
$$

where $\beta=4 x+3$

The smallest positive integer solution of the above equation is

$$
\beta_{0}=3, y_{0}=1
$$

and the general solution of equation (15) is given by

where

$$
\begin{aligned}
& y_{n}=\frac{g_{n}}{4 \sqrt{2}} \\
& \beta_{n}=\frac{f_{n}}{2} \\
& f_{n}=(3+2 \sqrt{2})^{n+1}+(3-2 \sqrt{2})^{n+1} \\
& g_{n}=(3+2 \sqrt{2})^{n+1}-(3-2 \sqrt{2})^{n+1}, n=0,2,4, \ldots
\end{aligned}
$$

By using (18) in (16) and using (2), we obtain the non-zero distinct integral solutions to (1) are given by

$$
\begin{aligned}
& x_{n}=\frac{f_{n}-6}{8} \\
& y_{n}=\frac{g_{n}}{4 \sqrt{2}} \\
& z_{n}=\frac{f_{n}+2}{8} \\
& w_{n}=x_{n}=\frac{f_{n}-6}{8}, n=0,2,4 \ldots
\end{aligned}
$$


On the Cubic Diophantine Equation with Four Unknowns

\subsection{Illustration 3}

Choosing $k=-h$ in (3), we obtain

$$
x^{2}+y^{2}=6 h x^{2}+2 h^{3}
$$

To solve (19), we have to take particular values of $h$.

For illustrations, taking $\mathrm{h}=4$, in (19), we have

$$
y^{2}=23 x^{2}+128
$$

By taking $y=2 Y$ and $x=2 X$, we get

$$
Y^{2}=23 X^{2}+32
$$

The smallest positive integer solution of (21) is

$$
X_{0}=4, Y_{0}=20
$$

To obtain the other solutions of (21), consider the pell equation

$$
Y^{2}=23 X^{2}+1
$$

whose general solution is given by

where

$$
\begin{aligned}
& \tilde{Y}_{n}=\frac{f_{n}}{2} \\
& \tilde{X}_{n}=\frac{g_{n}}{2 \sqrt{23}} \\
& f_{n}=(24+5 \sqrt{23})^{n+1}+(24-5 \sqrt{23})^{n+1} \\
& g_{n}=(24+5 \sqrt{23})^{n+1}-(24-5 \sqrt{23})^{n+1}, n=-1,0,1,2, \ldots
\end{aligned}
$$

Applying Brahmagupta lemma between $\left(X_{0}, Y_{0}\right)$ and $\left(\tilde{X}_{n}, \tilde{Y}_{n}\right)$, the other integer solutions of (21) are given by

$$
\begin{aligned}
& X_{n+1}=2 f_{n}+\frac{10}{\sqrt{23}} g_{n} \\
& Y_{n+1}=10 f_{n}+2 \sqrt{23} g_{n}
\end{aligned}
$$

By applying (23) in (20), we obtain the non-zero distinct integral solutions to (1) are given by

$$
\begin{aligned}
& x_{n+1}=4 f_{n}+\frac{20}{\sqrt{23}} g_{n} \\
& y_{n+1}=20 f_{n}+4 \sqrt{23} g_{n} \\
& z_{n+1}=4 f_{n}+\frac{20}{\sqrt{23}} g_{n}+4 \\
& w_{n+1}=4 f_{n}+\frac{20}{\sqrt{23}} g_{n}-4, n=-1,0,1,2, \ldots
\end{aligned}
$$

Following the analysis presented in Illustration 1, one may obtain relations among the solutions for Illustrations 2 and 3. 


\section{M.A.Gopalan and V.Krithika}

\section{Conclusion}

In this paper, we have presented sets of infinitely many non-zero distinct integer solutions to the cubic equation with four unknowns given by $x^{2}+y^{2}=z^{3}-w^{3}$. In other words, we have obtained quadruples such that, in each quadruple, the sum of the squares of any two members equals the difference of cubes of its other two members. As Diophantine equations are rich in variety due to their definition, one may attempt to find integer solutions to higher degree Diophantine equations with multiple variables along with their suitable properties.

\section{REFERENCES}

1. L.J.Mordell, Diophantine Equations, Academic press, New York, (1969).

2. R.D.Carmichael, The Theory of Numbers and Diophantine Analysis, New York, Dover, (1959).

3. L.E.Dickson, History of Theory of Numbers, vol.2, Diophantine Analysis, New York, Dover, (2005)

4. S.G.Telang, Number Theory, Tata Mc Graw Hill Publishing Company, NewDelhi (1996).

5. M.A.Gopalan, S.Vidhyalakshmi and A.Kavitha, Observations on the ternary cubic equation $x^{2}+y^{2}+x y=12 z^{3}$, Antarctica J. Math., 10(5) (2013) 453-460.

6. M.A.Gopalan and K.Geetha, On the ternary cubic diophantine equation, $x^{2}+y^{2}-x y=z^{3}$, Bessels J. Math., 3(2) (2013) 119-123.

7. S.Vidhyalakshmi, M.A.Gopalan and A.Kavitha, Observations on the ternary cubic equation $x^{2}+y^{2}-x y=7 z^{3}$, International Journal of Computational Engineering and Resarch, 3(5) (2013) 17-22.

8. M.A.Gopalan, S.Vidhyalakshmi, G.Sumathi, On the ternary cubic diophantine equation $x^{3}+y^{3}+z\left(x^{2}+y^{2}-20\right)=4(x+y)^{2} z$, Impact J. Sci. Tach, 7(2) (2013) 0106.

9. S.Vidhyalakshmi, T.R.Usha Rani, M.A.Gopalan, Integral solutions of nonhomogeneous cubic equation $a x^{2}+b y^{2}=(a+b) z^{3}$, Diophantine J.Math., 22(1) (2013) 31-38.

10. K.Meena, M.A.Gopalan, S.Vidhyalakshmi and S.Aarthy Thangam, On the ternary non-homogeneous cubic equation $4(x+y)^{2}-7 x y+(x+y)+15(x-y)=16\left(z^{3}-1\right)$, Bessel J. Math., 4(3) (2014) 75-80.

11. S.Vidhyalakshmi, M.A.Gopalan and A.Kavitha, On the ternary cubic equation $5(X+Y)^{2}-7 X Y+X+Y+1=23 z^{3}$, IJIRR, 1(10) (2014) 99-101.

12. M.A.Gopalan, N.Thirunraiselvi, V.Krithika, On the ternary cubic Diophantine equation $7 x^{2}-4 y^{2}=3 z^{3}$, International Journal of Recent Scientific Research, 6(9) (2015) 6197-6199.

13. M.A.Gopalan, S.Vidhyalakshmi and N.Thiruniraiselvi, On the Non-Homogeneous ternary Cubic Equation $2 a^{2}\left(x^{2}+y^{2}\right)-2 a(k+1)(x+y)+(k+1)^{2}=2^{2 n} z^{3}$, Universe of Emerging Technologies and Science, 2(1) (2015) 1-5. 
On the Cubic Diophantine Equation with Four Unknowns

14. M.A.Gopalan and K.Geetha, Observations on Cubic Equation with four unknowns $x^{3}+y^{3}+x y(x+y)=z^{3}+2(x+y) w^{2}$, International Journal of Pure and Applied Mathematical Sciences, 6(1) (2013) 25-30.

15. M.A.Gopalan, ManjuSomanath, and V.Sangeetha, Lattice points on Homogeneous cubic equation with four unknowns $(x+y)\left(x y+w^{2}\right)=\left(k^{2}-1\right) z^{3}, k>1$, Indian Journal of Science, 2(4) (2013) 97-99.

16. M.A.Gopalan, S.Vidhyalakshmi, T.R.UshaRani, Integral Solutions of cubic equation with five unknowns $x^{3}+y^{3}+u^{3}+v^{3}=3 t^{3}$, IJAMA, 4(2) (2012) 147-151.

17. M.A.Gopalan, S.Vidhyalakshmi and N.Thiruniraiselvi, On the cubic equation with five unknowns $3\left(x^{3}-y^{3}\right)=z^{3}-w^{3}+12 t^{2}+4$, International Journal of Engineering, Science and Mathematics, 2(1) (2013) 227-236.

18. M.A.Gopalan., etal., On the Cubic Equation with six unknowns $x^{3}+y^{3}+z^{3}+w^{3}=2\left(P^{3}+Q^{3}\right), \quad$ International Journal of Science and Technology, The Experiment, 18(1) (2014) 1218-1223.

19. M.A.Gopalan, N.Thiruniraiselvi and K.Agalya, Solutions of the homogeneous Cubic Equation with six unknowns $\left(w^{2}+p^{2}-z^{2}\right)(w-p)=\left(k^{2}+2\right)(x+y) R^{2}$, Jamal Academic Research Journal, i, (2016) 273-278. 